\title{
A Lattice Model for Colloidal Gels and Glasses
}

\author{
Florent Krzakala, ${ }^{1}$ Marco Tarzia, ${ }^{2}$ and Lenka Zdeborová ${ }^{3,4}$ \\ ${ }^{1}$ CNRS; ESPCI, 10 rue Vauquelin, UMR 7083 Gulliver, Paris, France 75005, PCT \\ ${ }^{2}$ Institut de Physique Théorique, Orme des Merisiers - CEA Saclay, 91191 Gif Sur Yvette Cedex, France \\ 3 Université Paris-Sud, LPTMS, UMR8626, Bât. 100, Université Paris-Sud 91405 Orsay cedex \\ ${ }^{4}$ CNRS, LPTMS, UMR8626, Bât. 100, Université Paris-Sud 91405 Orsay cedex
}

\begin{abstract}
We study a lattice model of attractive colloids. It is exactly solvable on sparse random graphs. As the pressure and temperature are varied it reproduces many characteristic phenomena of liquids, glasses and colloidal systems such as ideal gel formation, liquid-glass phase coexistence, jamming, or the reentrance of the glass transition.
\end{abstract}

PACS numbers: 64.70.pv,64.75.Xc,64.70.qd

There is a growing experimental and theoretical interest in the study of colloidal suspensions of mesoscopic particles immersed in a polymeric solution. Their properties can be controlled precisely via chemical or physical manipulations, and the inter-particle interaction tuned from a very short-range depletion-induced attraction to a long-range Coulombic repulsion 1]. This makes colloids important both in the terms of basic scientific research and industrial applications 2]. Colloidal suspensions also offer new scenarios of dynamically arrested structures, which are the main focus of this work.

At high densities these systems exhibit the same glassy dynamics as a system of hard spheres [3]. The lack of available space at densities $\rho \approx 0.58$ gives rise to a phase called repulsive glass. If a short-range attraction is present, ergodicity may also be broken, resulting in the so-called attractive glass. When the range of attraction is less than $\approx 10 \%$ of the sphere diameter, the interplay between these two mechanisms can lead to a remarkable reentrance of the glass transition line, with the (energetically driven) attractive glass dominating at low temperature and the (entropically driven) repulsive glass at high temperature. This reentrance was first predicted by the mode coupling theory and later confirmed by experiments and simulations [4]. Interestingly at low temperatures the attractive glass transition moves to much lower densities. Such a low-density arrested state is often identified as a colloidal gel [5] - a low-density disordered arrested state which does not flow but possess solid-like properties such as a yield stress. However, the dynamical arrest at low densities and its exact relation to the glass transition is still very poorly understood, and our work also sheds light on this aspect.

In the low density - low temperature regime, a system of hard-core particles with a short-range attraction undergoes a phase separation between a colloid-rich and a colloid-poor phase [6]. The interplay between phase separation and gelation is also a widely discussed topic. Typically, when quenching from high temperature, the phase separation boundary is crossed first. When the glass transition is crossed at lower temperatures, the spinodal decomposition induces the formation of dense arrested regions, a situation called arrested phase separation [7]. Another phenomenon can be obtained by reducing the phases-coexisting regions to even lower densities and temperatures, by decreasing the average coordination number of particles, as proposed in [8, 9]. Under these conditions the phase separation does not intervene in a wide region of densities and the system can be equilibrated by annealing down to very low temperatures, leading to a state of matter called ideal gel [10].

Theoretical progress has been hampered by the lack of microscopic models able to roughly locate and describe the colloidal gel transition. In this letter we discuss an exactly solvable yet realistic model characterized by valence-limited interactions that generalizes the lattice glass model introduced by Biroli and Mézard (BM) in [11]. The model is motivated by the classical shortrange depletion attraction but also by recent studies of a family of patchy [9] or limited-valency potentials [12]. It allows to reproduce the behavior of a broad variety of complex materials that are usually simulated at high computer cost. Under different conditions we observed in particular the liquid-glass coexistence, the reentrant glass transition and the formation of an ideal gel. This leads to an unifying description of the structural arrest in different amorphous and disordered systems, ranging from hard-spheres to colloids and gels.

The model - We consider a system of particles on a lattice. Each site $i$ carries $n_{i}=0$ or $n_{i}=1$ particles, with a short-range attractive interaction giving a unit energy gain to all pairs of nearest-neighbors particles. Motivated by the concepts of geometrical frustration and valencelimited interactions [8, 9, 12], we follow BM [1] and restrict the occupation by a hard constraint: a particle cannot have more than $\ell$ occupied neighbors. The partition function (where $\beta$ is the inverse temperature and $\mu$ the chemical potential) reads

$$
Z(\mu, \beta)=\sum_{\text {allowed }\{n\}} e^{\mu \sum_{i=1}^{N} n_{i}+\beta \sum_{<i j>} n_{i} n_{j}}
$$

where the sum is restricted over all configurations $\{n\}$ satisfying the hard constraints. For $\beta=0$, the attraction 
is turned off and the model reduces to the BM model [11]. In real (continuous) space, the presence of the maximum possible number of neighbors $l$ gives rise to an entropic loss 13]. This effect is, however, inhibited on the lattice and we thus also consider a variant of the model where each sites with exactly $\ell$ occupied neighbors induce a penalty $e^{-s_{p}}$ in eq. (11). This entropic repulsion is only relevant at large temperatures and is small compared to the attractive potential at low temperatures.

The solution - We shall focus on the exact solution of the model on large $c$-regular random graphs (where the number of neighborhooding sites, connectivity, of each vertex is exactly $c>l$ ). Such graphs are locally tree-like, i.e., there are no cycles up a distance scaling like $\log N$ from a typical site. The topological disorder and the geometrical frustration are introduced by very long loops that disfavor the crystalline order and let emerge more easily the glassy phases. This corresponds to a mean-field like approach to the problem 14].

In the low density-high temperature region the Bethe approximation gives an exact solution on tree-like lattices. For $s_{p}=0$ (but the generalization is straightforward) it is obtained from the fixed point of

$$
\begin{array}{r}
\psi_{e}=\frac{1}{Z_{0}}, \quad \psi_{s}=\frac{e^{\mu+\ell \beta}}{Z_{0}}\left(\begin{array}{c}
c-1 \\
\ell
\end{array}\right) \psi_{u}^{\ell} \psi_{e}^{c-1-l}, \\
\psi_{u}=\frac{e^{\mu}}{Z_{0}} \sum_{\ell^{\prime}=0}^{\ell-1} e^{\ell^{\prime} \beta}\left(\begin{array}{c}
c-1 \\
\ell^{\prime}
\end{array}\right) \psi_{u}^{\ell^{\prime}} \psi_{e}^{c-1-\ell^{\prime}},
\end{array}
$$

where $\psi_{e} / \psi_{s} / \psi_{u}$ are the probabilities that a node is empty/saturated/unsaturated when one of its neighborhooding sites is removed from the graph, $Z_{0}$ is a normalization constant ensuring that $\psi_{e}+\psi_{s}+\psi_{u}=1$. The free energy density $J=\log Z / N$ then reads

$$
\begin{aligned}
J(\mu, \beta) & =\log \left[1+e^{\mu} \sum_{\ell^{\prime}=0}^{l} e^{\ell^{\prime} \beta}\left(\begin{array}{c}
c \\
\ell^{\prime}
\end{array}\right) \psi_{u}^{\ell^{\prime}} \psi_{e}^{c-\ell^{\prime}}\right] \\
& -\frac{c}{2} \log \left[\psi_{e}^{2}+2 \psi_{e}\left(\psi_{u}+\psi_{s}\right)+e^{\beta} \psi_{u}^{2}\right] .
\end{aligned}
$$

The density of occupied sites is given by $\rho=\partial_{\mu} J(\mu, \beta)$.

As the density increases, or the temperature decreases, a dynamical glass transition arises at $\rho_{d}(\beta)$ where the free energy landscape divides into exponentially many valleys [15]. Each valley corresponds to a different Gibbs state $\alpha$ with a free energy density $J_{\alpha}$. This can be recognized from the divergence of the point-to-set correlation length, or, equivalently, by the emergence of a nontrivial glass solution described by the one-step replica symmetry breaking approach [15, 16, 17, 18]. The complexity $\Sigma(J)$ (sometime called configurational entropy) is defined as $\Sigma(J)=\log \mathcal{N}(J) / N$, where $\mathcal{N}(J)$ is the number of states with free energy density $J$. It can be computed via its Legendre transform $\Phi(m, \mu, \beta)$ defined by

$$
e^{N \Phi(m, \mu, \beta)} \equiv \sum_{\alpha} e^{m N J_{\alpha}(\mu, \beta)}=\int \mathrm{d} J e^{N[m J(\mu, \beta)+\Sigma(J)]},
$$

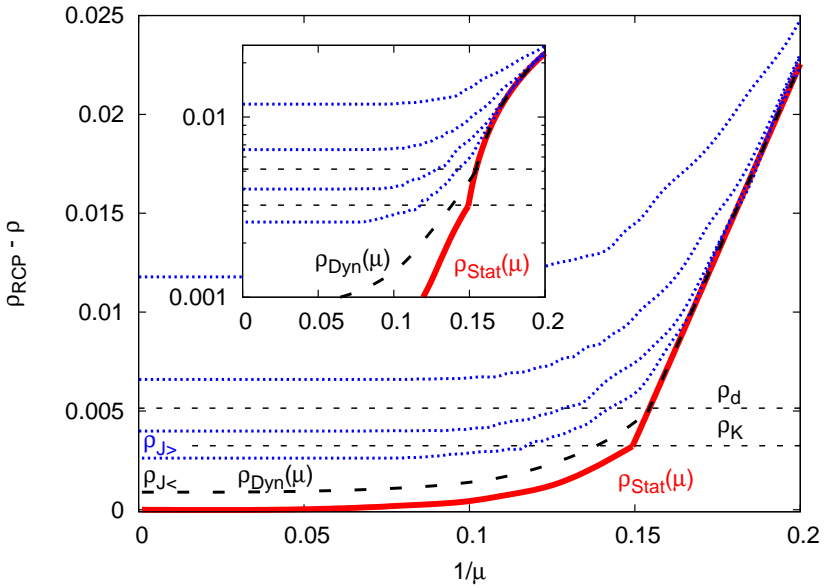

FIG. 1: (color online) High temperature behavior of the model with $c=3, l=1$ and no entropic penalty. $\rho_{\mathrm{RCP}}-\rho$ is the difference between the packing fraction, or density, and its maximum achievable value (random close packing), $\mu$ is the chemical potential. The static equilibrium solution (bold/red) undergoes a phase transition at $\rho_{K}=0.5725$ and converges to the densest packing $\rho_{\mathrm{RCP}}=0.57574$ at $\mu=\infty$. However, the dynamics falls out of equilibrium at $\rho_{d}=0.5708$ and the packing fraction following a slow annealing can be increased up to at most $0.5731 \leq \rho_{J} \leq 0.5748$. The dotted/blue curves are Monte-Carlo annealings for $N=10^{5}$ with rates $d \mu=10^{-4}, 10^{-5}, 10^{-6}$ and $10^{-7}$ (from top to bottom) and the (dashed/black) line $\rho_{\text {Dyn }}$ is the iso-complexity approximation for the infinitely slow annealing (see the text). The inset shows the same plot on a logarithmic scale.

where the sum runs over all the different Gibbs states $\alpha$ and the Legendre parameter $m$ is equivalent to the Parisi parameter in the replica theory [15, 19]. The cavity method derives a set of self-consistent equations for $\Phi(m, \mu, \beta)$ 15. They can be solved analytically in the limit $\mu \rightarrow \infty$ [11]. In general, however, these equations are solved using the numerical population dynamics technique of 15. The case $m=1$ also allows significant simplification [17]. We also checked self-consistently, using the method of [18], that the one-step replica symmetry breaking solution is stable, and the presented equilibrium solution is thus generally considered exact.

The glass and jamming transitions - We first revisit the behavior the of the model in the high temperature limit $(\beta=0)$ where it reduces to the original $\mathrm{BM}[11]$, and consider the case $c=3, \ell=1$ for concreteness. FIG. 1] depicts in bold/red the difference between the largest possible density of occupied sites $\rho_{\mathrm{RCP}}$ and the equilibrium density $\rho$ as a function of the chemical potential $\mu$. In the equilibrium solution, the model undergoes a Kauzmann transition at $\rho_{K}=0.5725$ where the complexity vanishes and the system becomes a an ideal glass (a "static" glass phase) 14]. The density of the glass can be in principle further increased in the equilibrium solution, and the densest possible packing is reached at $\rho_{\mathrm{RCP}}=0.57574$ (Random Close Packing, first computed in [11].) 

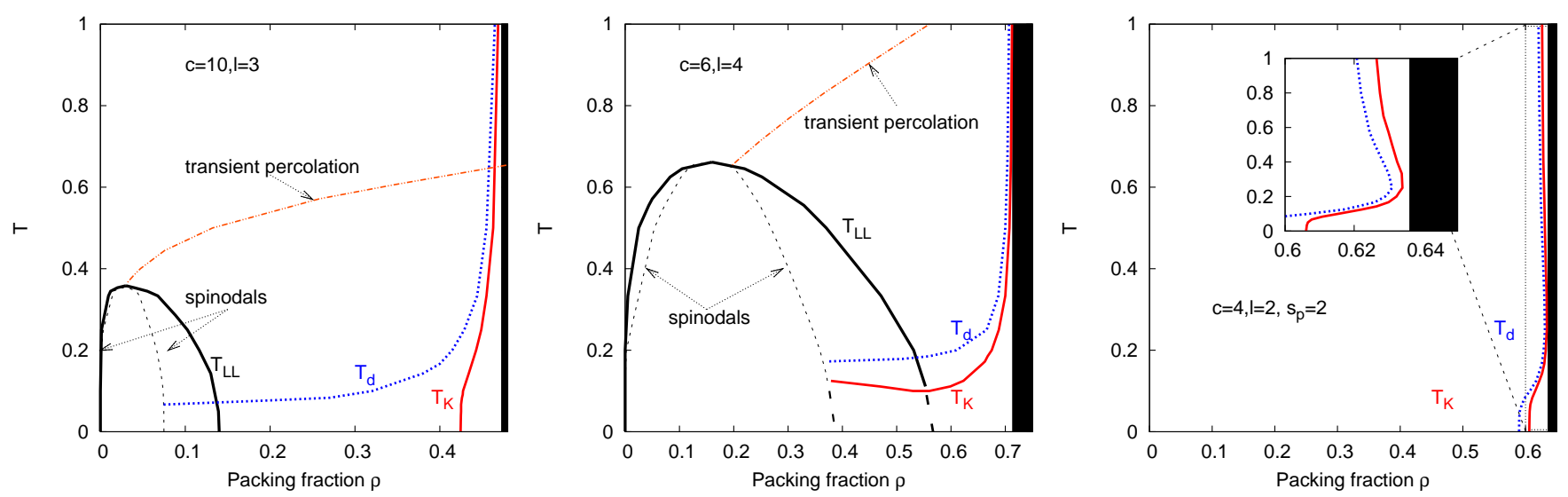

FIG. 2: (color online) Three representative phase diagrams of the model in the temperature $(T)$ - packing fraction $(\rho)$ plane. The black/continuous curves are the colloid-rich/colloid-poor coexistence lines $T_{\mathrm{LL}}$, and the corresponding spinodal lines are black/dashed curves. The orange/dashed-dotted curves correspond to the percolation of physical clusters, and intercept the critical point of the phase separation. The dynamical $T_{d}(\rho)$ (blue/dotted curve) and the Kauzmann $T_{K}(\rho)$ (red/continuous curve) glass transition lines are also shown. The black filled area is beyond the random close packing fraction $\rho_{\mathrm{RCP}}$. Left panel: $c=10, l=3$ and no entropic penalty. An ideal gel appears at low enough temperature $(T \lesssim 0.1)$ and low volume fraction $(0.1 \lesssim \rho \lesssim 0.4)$. Central panel: $c=6, l=4$ and no entropic penalty. A phase coexistence between a colloid-poor and an attractive colloid-rich glass phase occurs for low temperature $(T \approx 0.05)$ resulting in an arrested phase separation. Right panel: $c=4, l=2$ and $s_{p}=2$. The glass transition lines $T_{d}(\rho)$ and $T_{K}(\rho)$ exhibit a reentrant shape (see inset), resulting in a glass-liquid-glass transition along isochores at densities around $\rho \approx 0.62$.

The dynamical behavior is more involved 20]. The equilibration time diverges (as a power law) at the dynamical transition $\rho_{d}=0.5708<\rho_{K}$ where the system is trapped in one of the exponentially many Gibbs states and falls out-of-equilibrium. When the pressure is further increased the system evolves within this state and the density can again be further increased [21], but does not follow the equilibrium curve. It is not yet known how to describe analytically this process on random graphs, and in FIG. 1 we thus display the results of Monte Carlo annealings at different rates. The slowest annealing we tried reached $\rho \approx 0.5731$. We also computed the isocomplexity approximation curve for an infinitely slow annealing, where the dynamics is assumed to follow a curve defined by $\Sigma\left(\rho_{\text {Dyn }}(\mu)\right)=\Sigma\left(\rho_{d}\right)$ [22]. This actually yields a lower bound on the infinite pressure density reached by this procedure. In our model, the density reached by an infinitely slow annealing, $\rho_{J}$, is the analog of the Jamming point [23] and we thus obtain $0.5731 \geq \rho_{J} \geq 0.5748$. Note that, as discussed in [21], $\rho_{J}$ does not correspond to the densest possible packing $\rho_{\mathrm{RCP}}$ neither to the dynamical or Kauzmann density $\rho_{d}$ or $\rho_{K}$. In finite dimensional systems the dynamical transition is smeared out by activation processes that restore the ergodicity and prevent a complete freezing of the system [14]. In that case, an ideal glass transition (if any) should thus arise only at the Kauzmann point $\rho_{K}$.

Phase diagrams - We now discuss the different phase diagrams obtained by varying the connectivity $c$, the maximum number of occupied neighbors $\ell$, and the entropic penalty $s_{p}$. Three representative situations are depicted in FIG. 2.

The left panel of FIG. 2 2 shows a typical phase diagram when the valency restriction $l$ is small (but larger than 2 ) and the dimension-like parameter $c$ is large. In this situation a colloid-rich/colloid-poor phase coexistence is located in a narrow region of small temperatures $T$ and densities $\rho$, in agreement with [9]. The dynamical glass transition line $T_{d}$ then bends towards low value of $\rho$ and continues in an almost horizontal direction. Under these conditions dynamical arrest phenomena and glassy behavior should emerge at very small volume fractions. This state of matter is as close as possible to an ideal gel [9, 10] which is in our model equivalent to a glass in the dynamical phase. In contrast, the Kauzmann glass transition line $T_{K}$ typically stays almost vertical in the $T$ - $\rho$ plane: a true equilibrium glass transition thus only takes place at high densities. In a finite dimensional system, where the dynamical transition is smeared out, the dynamics along isochores should follow approximatively an Arrhenius dependence, exactly as found in [8, 9]. Interestingly enough, the percolation line is located much above the dynamic arrest (gel) one. This gap is due to finite life time of bonds at finite temperature, this is common for colloidal gelation. Nevertheless, the formation of a spanning cluster could affect the dynamical behavior of the system, as shown in [24].

The central panel of FIG. 2 shows a typical phase diagram when the valency restriction $l$ is large and $c$ small. The coexistence region is much broader with respect to the previous case. And both the dynamical and the Kauzmann transition lines bend towards low densities. 
An ideal glass state thus emerges for values of $\rho$ much smaller than $\rho_{\mathrm{RCP}}$. The glass transition lines moreover intercept the spinodal so that a deep quench of the system at low enough temperatures (e.g., $T \approx 0.05$ ) into the coexistence region (e.g., $\rho \approx 0.2$ ) results in a phase separation between a colloid-poor and a glassy colloidrich region. Under these conditions the phase separation process is interrupted by the formation of an (attractive) glass in the dense phase. This arrested phase separation scenario is one of the possible routes to colloidal gelation and the formation of a low density gel state [7].

The right panel of FIG. 2 shows a case with the valency restricted to $l=2$. In this case the occupied sites can create only chains and the phase separation region is absent, this was also observed in [9]. A more interesting feature shown in this diagram is the reentrant shape of the dynamical and the Kauzmann transition lines. The reentrant behavior can be observed when the short-range attractive potential is in competition with the entropic repulsion, $s_{p}$, in agreement with [4]. At large temperatures the interaction contributing to the formation of a glass is the entropic repulsion $s_{p}$, at low temperature it is the attractive potential. Note that the reentrant behavior is usually observed together with the presence of the phase separation region, and we indeed observe the two effects in the left and central panel if we set the entropic repulsion $s_{p}$ to a positive value. Our example shows however that the reentrant behavior is not necessarily connected with the phase separation, nor with a presence of a glass-glass transition, in agreement with earlier works [4, 25].

Conclusions - We have studied a lattice model of complex fluids, which can be solved exactly on random graphs using the cavity method [15]. Despite its intrinsic simplicity, it captures many features that arise in real systems and it illustrates physical properties of arrested states observed in colloidal suspensions under different conditions. It reproduces the shape of a variety of phase diagrams, allowing for a qualitative comparison with experiments. In the future, it should be interesting to study the coarsening dynamics associated to the phase separation between the colloid-poor and the colloid-rich phase and to simulate the dynamics in finite dimension, as in [11, 26, 27]. It is also of interest to understand if a glassglass transition can be found for this class of models.

We thank A. Coniglio, J. Kurchan, O. Rivoire, T. Mora, F. Zamponi and E. Zaccarelli for discussions.

[1] A. Yethiraj and A. V. Blaaderen, Nature 421, 513 (2003).

[2] V. Trappe et al., Nature 411, 772 (2001); F. Sciortino and P. Tartaglia, Advances in Physics 54, 471 (2005); W. B. Russel et al., Colloidal Dispersions (Cambridge University Press, Cambridge, 1991).

[3] P. N. Pusey and W. van Megen, Phys. Rev. Lett. 59,
2083 (1987).

[4] K. A. Dawson et al., Phys. Rev. E 63, 011401 (2001); K. Pham et al., Science 296104 (2002); G. Foffi et al., Phys. Rev. E 65, 050802 (2002). S. Chen et al., Science 300, 619 (2003).

[5] H. Verduin and J. Dhont, J. Coll. Int. Sci. 172, 245 (1995); N. Verhaegh et al., Physica A 242, 104 (1997). S. A. Shah et al., J. Phys.: Condens. Matter 15, 4751 (2003); P. N. Segrè et al., Phys. Rev. Lett. 86, 6042 (2001).

[6] Hill T L, An Introduction to Statistical thermodynamics, New York: Dover, 1987.

[7] P. J. Lu et al, Nature 453, 499-503 (2008).

[8] E. Del Gado and W. Kob, Europhys. Lett. 72, 1032 (2005); ibid, Phys. Rev. Lett. 98, 028303 (2007).

[9] E. Zaccarelli et al., J. Chem. Phys. 124, 124908 (2006); E. Bianchi et al., Phys. Rev. Lett. 97, 168301 (2006). S. Sastry et al., J. Stat. Mech. P12010 (2006).

[10] F. Sciortino et al.. Computer Physics Communications 169 (2005) 166-171. E. Zaccarelli, J. Phys.: Condens. Matter 19, 323101 (2007).

[11] G. Biroli and M. Mézard, Phys. Rev. Lett. 88, (2002) 025501. O. Rivoire et al., Eur. Phys. J. B 37, 55 (2004).

[12] R. J. Speedy and P. G. Debenedetti, Mol. Phys. 81, 237 (1994).

[13] G. Tarjus et al., J. Phys.: Condens. Matter 15, 1077 (2003).

[14] T. Kirkpatrick and D. Thirumalai, Phys. Rev. Lett. 58, 2091 (1987); T. Kirkpatrick, P. Wolynes, Phys. Rev. A 35, 3072 (1987). M. Mézard and G. Parisi, Phys. Rev. Lett. 82, 747 (1999). G. Parisi and F. Zamponi, J. Chem. Phys. 123, 144501 (2005).

[15] M. Mézard and G. Parisi, Eur. Phys. J. B 20 (2001) 217.

[16] A. Montanari and G. Semerjian, J. Stat. Phys. 124, 103 (2006). J.-P. Bouchaud and G. Biroli, Phys. Rev. B 72, 064204 (2005).

[17] M. Mézard and A. Montanari, J. Stat. Phys. 1241317 (2006). F. Krzakala et al., Proc. Natl. Acad. Sci. 104, 10318 (2007).

[18] F. Krzakala and L. Zdeborová, Europhys. Lett. 81 (2008) 57005. L. Zdeborová and F. Krzakala, Phys. Rev. E 76 (2007) 031131.

[19] R. Monasson, Phys. Rev. Lett. 75, 2847 - 2850 (1995).

[20] J .P. Bouchaud et al. in Spin Glasses and Random Field, P. Young ed., World Scientific, Singapur (1997). L. F. Cugliandolo, in Les Houches Session 77, J-L Barrat, J Dalibard, J Kurchan, M V Feigel'man eds (2002).

[21] F. Krzakala and J. Kurchan, Phys. Rev. E 76, 021122 (2007).

[22] A. Montanari and F. Ricci-Tersenghi, Phys. Rev B 70, 134406 (2004).

[23] B. Lubachevsky and F. Stillinger, J. Stat. Phys. 60, 561 (1990). A. Liu and S. Nagel, Nature 396, 21 (1998).

[24] A. Coniglio et al., J. Phys.: Condens. Matter 16, S4831 (2004); E. Del Gado, A. Fierro, L. de Arcangelis, and A. Coniglio, Phys. Rev. E 69, 051103 (2004).

[25] G. Foffi et al Phys. Rev. Lett. 90, 238301 (2003), P. Kumar et al. Phys. Rev. E 72, 021501 (2005)).

[26] M. Pica Ciamarra et al., Phys. Rev. E 67, 057105 (2003).

[27] E. Marinari and V. Van Kerrebroeck, Europhys. Lett. 73, 383 (2006), and G. D. McCullagh et al., Phys. Rev. E 71, 030102(R) (2005). 